\title{
Challenges in recruiting children to a multidrug-resistant TB prevention trial
}

\author{
S. Purchase, ${ }^{1}$ E. Batist, ${ }^{1}$ N. Mmile, ${ }^{2}$ S. Nkosi, ${ }^{3}$ J. Workman, ${ }^{1}$ N. Martinson, ${ }^{2}$ L. Fairlie, ${ }^{3}$ H. S. Schaaf, ${ }^{1,4}$ \\ L. Choo, ${ }^{5}$ C. McGowan, ${ }^{5}$ A. M. Crook, ${ }^{5}$ J. A. Seddon, ${ }^{1,6}$ A. C. Hesseling ${ }^{1}$ \\ 1Desmond Tutu TB Centre, Department of Paediatrics and Child Health, Faculty of Medicine and Health Sciences, \\ Stellenbosch University, Tygerberg, 2Perinatal HIV Research Unit, University of Witwatersrand, Johannesburg, \\ ${ }^{3}$ Wits Reproductive Health \& HIV Institute, Faculty of Health Sciences, University of the Witwatersrand, \\ Johannesburg, ${ }^{4}$ Department of Paediatrics and Child Health, Faculty of Medicine and Health Sciences, Stellenbosch \\ University, Cape Town, South Africa; 5 Institute of Clinical Trials and Methodology, MRC Clinical Trials Unit at \\ University College London, London, ${ }^{6}$ Department of Infectious Diseases, Imperial College London, Norfolk Place, \\ London, UK
}

S U M M A R Y

BACKGROUND: Recruitment to randomised clinical trials can be challenging and slow recruitment has serious consequences. This study aimed to summarise and reflect on the challenges in enrolling young children to a multidrug-resistant TB (MDR-TB) prevention trial in South Africa.

METHODS: Recruitment to the Tuberculosis Child Multidrug-resistant Preventive Therapy Trial (TBCHAMP) was tracked using an electronic recruiting platform, which was used to generate a recruiting flow diagram. Structured personnel questionnaires, meeting minutes and workshop notes were thematically analysed to elucidate barriers and solutions.

RESULT: Of $3,682(85.3 \%)$ adult rifampicin (RIF) resistant index cases with pre-screening outcomes, $1597(43.4 \%)$ reported having no children under 5 years in the household and $562(15.3 \%)$ were RIFmonoresistant. More than nine index cases were prescreened for each child enrolled. Numerous barriers to recruitment were identified. Thorough recruitment planning, customised tracking data systems, a dedicated recruiting team with strong leadership, adequate resources to recruit across large geographic areas, and excellent relationships with routine TB services emerged as key factors to ensure successful recruitment.

CONCLUSION: Recruitment of children into MDR-TB prevention trials can be difficult. Several MDR-TB prevention trials are underway, and lessons learnt from TB-CHAMP will be relevant to these and other TB prevention studies.

KEY WORDS: fluoroquinolone; TB-CHAMP; randomised controlled trial
Modelling data suggest that 2 million children globally are infected with multidrug-resistant TB (MDR-TB) (i.e., Mycobacterium tuberculosis resistant to isoniazid [INH] and rifampicin [RIF] with or without resistance to other drugs), with 25,00032,000 children progressing to MDR-TB disease each year. ${ }^{1,2}$ The recent call to action by the UN HighLevel Meeting included scaling up TB preventive therapy $(\mathrm{TPT})^{3}$ and the WHO has emphasized the need for high-quality evidence from placebo-controlled trials to inform policy. ${ }^{4}$ The Tuberculosis Child Multidrug-resistant Preventive Therapy Trial (TB-CHAMP) is a community-based, multi-site cluster-randomised, placebo-controlled trial to assess the efficacy and safety of 24 weeks of daily

JAS and ACH contributed equally levofloxacin in children aged $<5$ years exposed in their household to adults with MDR-TB. The trial is being implemented in South Africa and is the only trial exclusively investigating MDR-TB preventive therapy in young children.

Recruiting to randomised controlled trials (RCTs) can be challenging. A review of 440 trials between 2002 and 2008 found that only 55\% met their planned sample size, and almost half $(45 \%)$ had to request an extension. ${ }^{5}$ In a review of over 1000 RCTs, Kasenda and colleagues noted that a quarter were prematurely discontinued, with the most common reason for discontinuation being poor recruitment. ${ }^{6}$

There is a substantial body of literature highlighting common recruitment challenges to trials. Funding barriers include insufficient initial funding to cover recruiting costs and lack of additional funding for

Correspondence to: Susan Elizabeth Purchase, Desmond Tutu TB Centre, Department of Paediatrics and Child Health, Faculty of Medicine and Health Sciences, Francie van Zijl Drive, Stellenbosch University, Tygerberg 7505, Cape Town, South Africa. email: purchase@sun.ac.za / susanpurchase@gmail.com

Article submitted 18 February 2021. Final version accepted 17 May 2021. 
recruitment extention. ${ }^{7}$ Generally, the more rigorous the trial design, the more likely it is to encounter challenges. Strict inclusion and exclusion criteria, random allocation, blinding and use of placebo all make trials more challenging to enrol. ${ }^{7-9}$ Trials investigating infectious diseases in lower- and middle-income countries (LMICs) are usually conducted in poorer communities, with higher likelihood of migratory populations, violence, unemployment and substance abuse. Such trials are often conducted over large geographic areas, with poor public transport adding to recruitment complexity.

The lack of a sufficiently large pool of eligible participants to reach recruitment targets is another potential barrier. Muench's Third Law states that the estimated number of potential participants that can be recruited should be divided by 10 to get a more accurate forecast. ${ }^{10}$ Schulz and colleagues use a rule of $\pi$ by estimating how long recruitment will take and then multiplying by 3.14. For trials in LMICs, a multiplier of $2 \pi$ is recommended. ${ }^{10}$

Barriers to recruitment for trial participants may include fear of adverse effects, mistrust regarding research, logistical issues, severe illness, language or cultural barriers and stigma around the disease being researched. ${ }^{11}$ In paediatric prevention trials, caregivers may be reluctant to expose their well children to an experimental regimen. ${ }^{12,13}$

Consequences of poor recruitment can be dire, and include trial abandonment, reduced statistical power, the need for supplemental funding which diverts resources from other trials, and frustration for funders, researchers, participants and communities. Slow accrual may delay identification of potentially lifesaving interventions. ${ }^{10}$ There is limited literature regarding challenges to recruitment in TB trials, and almost no data regarding $\mathrm{TB}$ prevention or paediatric trials. Few trials even report on recruitment details. ${ }^{7}$ Three MDR-TB prevention trials are currently underway (ACTRN12616000215426; ${ }^{14}$ NCT03568383;15 ISRCTN92634082 ${ }^{16}$ ).

The purpose of this article is to describe challenges and solutions to recruitment in TB-CHAMP and provide practical lessons for investigators and other stakeholders to optimise recruitment to TB prevention trials, especially in children.

\section{METHODS}

\section{Study design}

This was a mixed-methods sub-study, combining a quantitative analysis of recruitment flow using a CONSORT diagram with a descriptive qualitative, reflective process evaluation of trial recruitment. The data presented were collected between 1 September 2017 (trial opened) and 31 July 2019 (when recruitment was temporarily paused due to funding challenges). We summarise the recruitment flow, and our reflections on the recruitment process and the solutions put in place to address several challenges faced across sites. The COVID-19 pandemic has added multiple layers of complexity to this trial, which we will report on later. Figure 1 shows participant flow until child randomisation.

\section{Trial management and approvals}

Stellenbosch University, Tygerberg, South Africa, is the trial sponsor, and trial management is supported by the Medical Research Council Clinical Trials Unit at University College, London, UK. The trial was approved by all relevant Research Ethics Committees or Institutional Review Boards in South Africa and the United Kingdom and by all required regulatory authorities. Index cases provided informed consent for their $\mathrm{TB}$ information to be captured and their household to be approached; caregivers provided informed consent for children's participation.

\section{Setting}

TB-CHAMP is being conducted at three South African research sites (Figure 2), all serving poor communities with a high burden of TB and HIV. Statistics from the Living Conditions Survey 2015 indicate that approximately half of South African adults live below the upper-bound poverty line, with $8 \%$ of children experiencing regular hunger. ${ }^{17}$

\section{Desmond Tutu TB Centre, Stellenbosch University,} Tygerberg, South Africa

Recruitment took place from more than 100 primary healthcare (PHC) clinics and nine hospitals across metropolitan Cape Town, Western Cape Province. The study opened with permission to work in a subset of these facilities; further permissions were sought when recruitment was slower than anticipated. Community members are highly migratory, and families often separated, with children living in the more rural neighbouring Eastern Cape Province.

\section{Perinatal HIV Research Unit, Matlosana, South Africa}

This peri-urban site is located at a general hospital, which serves as a referral centre for complicated MDR-TB patients from the North West Province. Recruitment took place from this hospital and three others with specialised MDR-TB units, and their referring PHC clinics. Most participants lived in relatively rural settings.

\section{Wits Reproductive Health and HIV Institute, Shandukani Research Centre, South Africa}

Children were recruited from 20 PHC clinics and four hospitals in Johannesburg and surrounds. Participants were from a variety of urban communities.

In Cape Town when the study opened, it was policy 


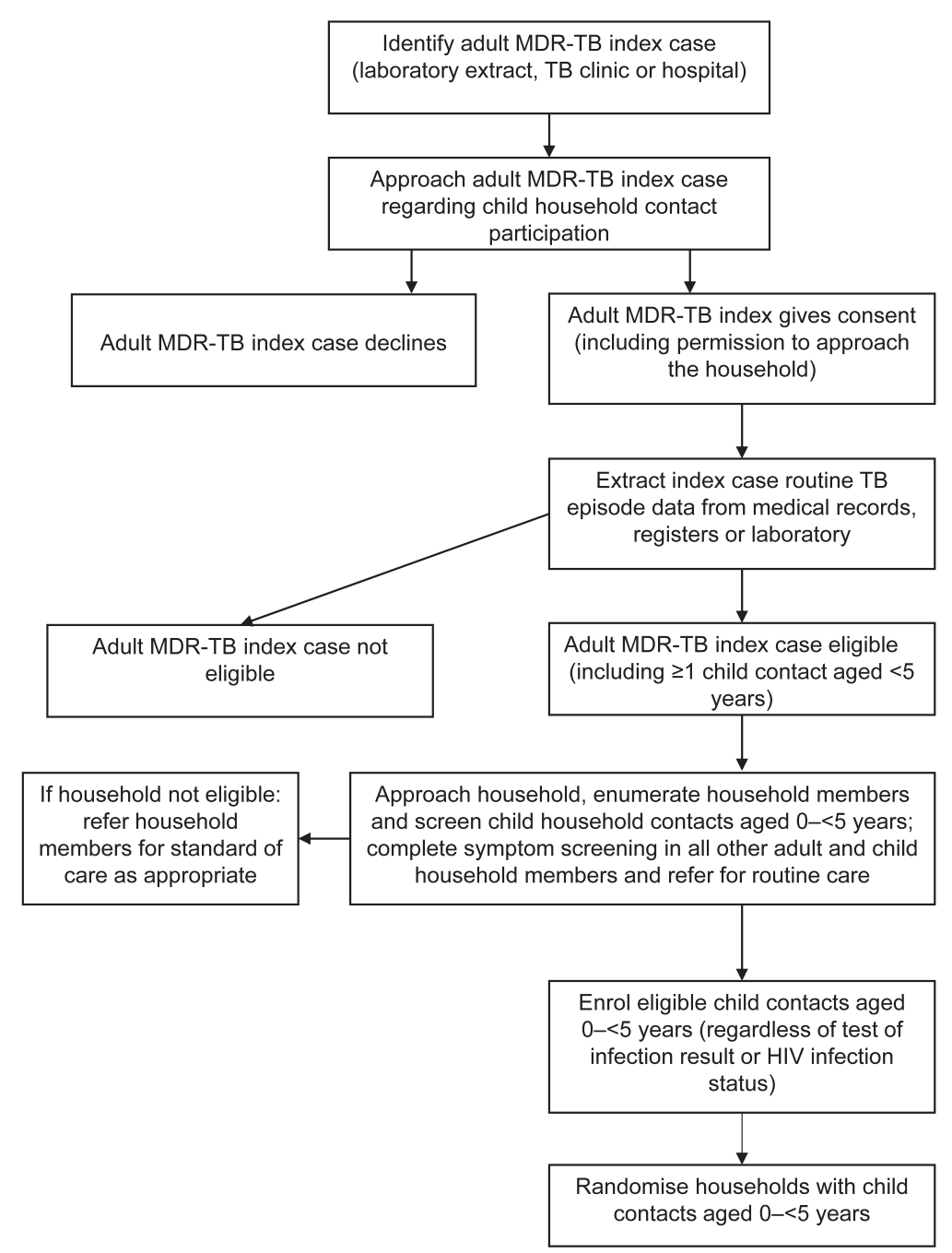

Figure 1 TB-CHAMP recruiting flow diagram. MDR-TB = multidrug-resistant TB; TB-CHAMP = Tuberculosis Child Multidrug-resistant Preventive Therapy Trial.

in routine care to identify and screen MDR-TB contacts below 5 years, with an option to offer a three-drug preventive therapy regimen. There was limited MDR-TB contact tracing implemented at the Matlosana and Shandukani sites.

\section{Recruiting strategies}

Adult MDR-TB index cases are identified from weekly data extracts of positive RIF-resistant Xpert $^{\circledR}$ MTB/RIF (Cepheid, Sunnyvale, CA, USA) results from the routine National Health Laboratory Service (NHLS), or from referrals by healthcare workers in routine $\mathrm{TB}$ services. Laboratory extracts were screened to assess initial eligibility. Permission was provided to the study teams to access this routine laboratory surveillance data to alert clinic personnel regarding potentially eligible index cases; the study team did not approach individual adult MDR-TB patients directly. Recruiting personnel assessed potential eligibility of the household and then arranged to obtain informed consent from the index case, typically at the clinic. Informed consent was also obtained from the caregiver/parent of the child participant. Potential child participants were screened at the trial sites for possible enrolment. This approach of starting with the adult MDR-TB index cases was necessary to ensure adequate identification and enrolment of child contacts, given the limited resources in routine $\mathrm{TB}$ services to conduct contact investigation in South Africa.

Each site developed its own recruiting plan and team structure, specific to the needs and challenges of its setting. Recruitment strategies were regularly revised with team structures altered and additional recruiters employed, with drivers functioning as recruiters and teams travelling up to $250 \mathrm{~km}$ from the research site to recruit participants.

Community advisory boards provided input into the study design, informed consent, and study messaging for the TB-CHAMP trial. The study team engaged regularly with routine healthcare services and study communities using posters, flyers and 


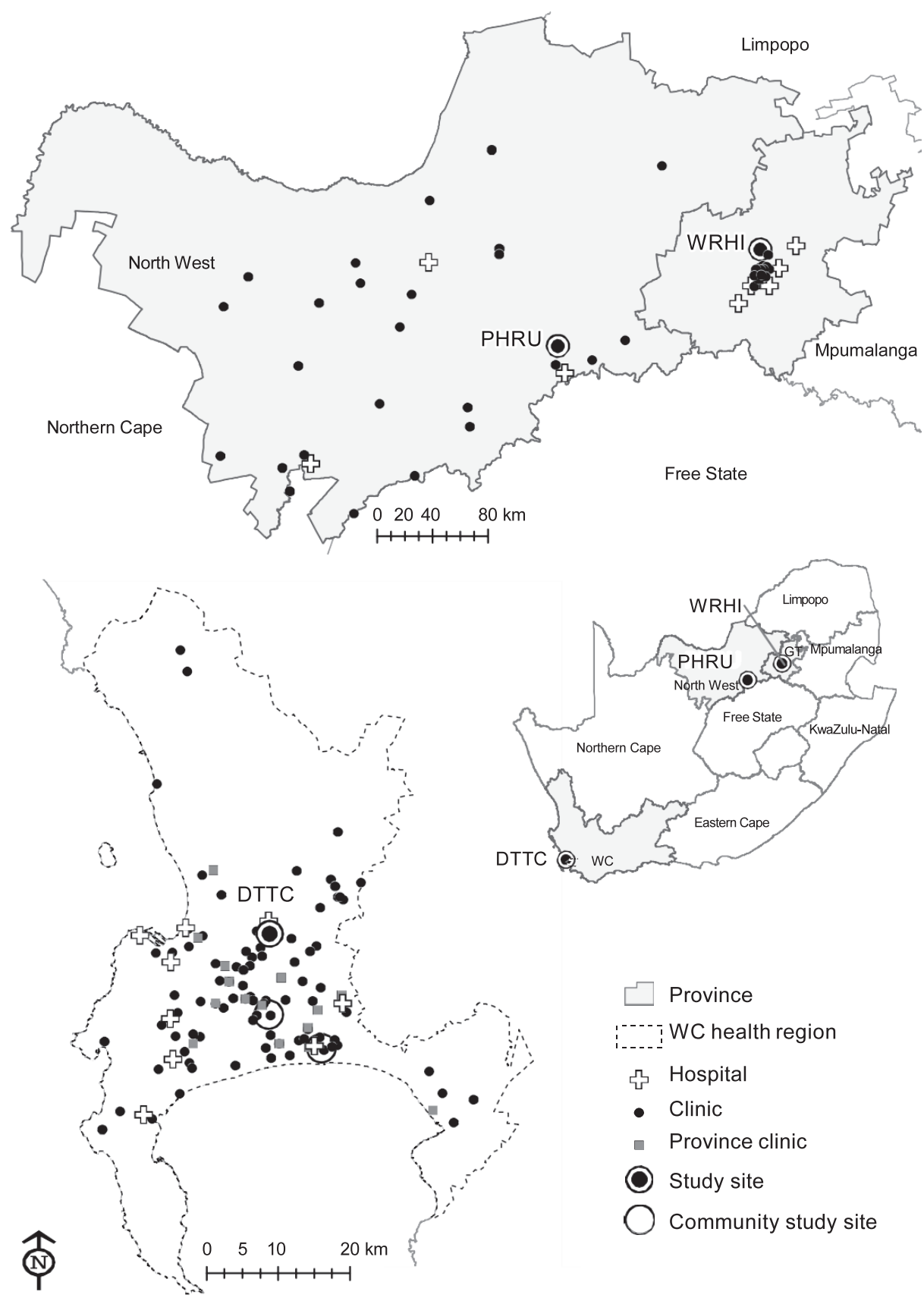

Figure 2 Location of South African sites conducting the TB-CHAMP study. PHRU = Perinatal HIV Research Unit, Soweto; WRHI = Wits Reproductive Health \& HIV Institute, Johannesburg; GT = Gauteng; DTTC = Desmond Tutu TB Centre, Cape Town; WC = Western Cape; TB-CHAMP = Tuberculosis Child Multidrug-resistant Preventive Therapy Trial.

regular visits to all clinics, and by attending local training and dissemination meetings.

\section{Tracking the recruitment process}

Pre-screening and screening processes were initially captured on paper-based logs. At two sites, online, shared spreadsheets were also used to track recruitment efforts. As numbers grew, these spreadsheets were found to be inefficient. A dedicated in-house recruiting platform "Mobilize" was developed to alleviate the administrative burden associated with recruitment tracking and to allow for accurate, upto-date feedback for the recruiting team. Data were stored in a SQL database on secure servers with restricted access. Recruiters accessed the data and managed their recruitment strategies daily using a REDCap (Vanderbilt University, Nashville, TN, USA) user-interface, and study leaders were able to view summary statistics and trends in a Microsoft PowerBI dashboard (Microsoft, Redmond, WA, USA), embedded on the access-controlled SharePoint page. 


\section{Data sources}

Data were drawn from "Mobilize", paper-based logs and the TB-CHAMP clinical trial CACTUS database to understand patient flow and drop-offs. Information used to identify barriers and solutions came from several sources. Weekly site meetings and monthly team calls were held to discuss recruitment challenges. Two questionnaires were administered to study personnel during 2018. The first, developed with input from a socio-behavioural specialist, was a structured questionnaire completed by each site, including 29 questions regarding overall recruitment strategy and specific challenges faced while recruiting index cases and children. The second questionnaire was completed individually by each study team member, and asked team members to describe in free text and in detail the three biggest recruitment challenges experienced. Recruiting teams drew on daily study diaries, highlighting challenges encountered in the field. A full-day in-person workshop and brainstorming session including team members from all sites was held, and written input from all teams regarding recruiting challenges and solutions was solicited and collated. Content analysis of questionnaires and meeting minutes was completed, and major themes and sub-themes were identified, analysed and collated.

\section{RESULTS}

\section{Recruitment outcomes}

There were 4,317 MDR-TB index cases identified overall over the 23-month recruiting period, mostly from the NHLS laboratory surveillance system (Figure 3). Of these, only 3,682 (85.3\%) had prescreening outcomes allocated on "Mobilize" - prescreening outcomes were not captured initially at all sites. Of the 3,682 index cases with outcomes, 1,597 $(43.4 \%)$ had no children under 5 years in the house and $562(15.3 \%)$ were excluded due to RIF monoresistance on line-probe assay. This figure does not accurately reflect levels of RIF monoresistance, as some index cases with monoresistance may have been screened out for other reasons, and in some cases study teams could not wait for phenotypic INH drug susceptibility test results before excluding index cases. The team was unable to contact 268 (7.3\%) index cases, despite multiple attempts. Forty-nine $(1.3 \%)$ index cases had already died by the time the team made contact. Of 3,682 index cases with outcomes, $298(8.1 \%)$ consented, allowing for the screening of 496 child contacts, with 450 children enrolled. Only $49(1.3 \%)$ index cases and $21(0.6 \%)$ caregivers refused consent; 1.5 children were enrolled from each index case recruited. Figure 3 shows the large number of index cases (9.6) needed to be pre- screened to recruit one child participant below 5 years of age.

\section{Recruiting challenges and solutions}

Tables 1-3 give the challenges and solutions to recruitment identified by the trial teams at sites. These included participant-, study- and team/resource-related challenges.

\section{Participants}

It soon became clear that study teams were dealing with households in crisis. Many index cases were hard to contact due to migration, hospitalisation or lack of a fixed address. Significant resources were expended for multiple clinic and home visits. Overall, $71 \%$ of index cases were either the primary caregiver or regularly cared for the child, and their illness impacted on family function. Anecdotally, perceived stigma related to TB and MDR-TB seemed prevalent, and study teams were often asked to visit homes in unmarked clothes and vehicles. Some individuals feared eviction from their homes should their MDR-TB status become public knowledge. Caregivers were hard to contact, often working outside of the home during the research teams' working hours. However, once contacted, few index cases and caregivers refused consent. The use of well-trained research assistants from local communities, obtaining informed consent in simple language and in the participant's preferred language was felt to be useful.

\section{Study design and setting}

TB-CHAMP is a complex trial with a long followup period. The rationale of the trial needed to be carefully explained in appropriate language, and the informed consent process was time-consuming. Dual written consent was needed (from the index case and the caregiver), which prolonged the consenting process. Locating index cases was challenging, partly due to the decentralised policy for treatment of MDR-TB in South Africa. There were far fewer under-5-year-old child contacts than the 2 per household that we anticipated based on data from previous observational MDR-TB studies. ${ }^{18,19}$ RIF monoresistance impacted recruitment and rates were somewhat higher than the $8-12 \%$ levels anticipated from 2007-2009 surveillance data accessed when the study was designed in 2012. ${ }^{20,21}$ We did not consider the impact of discordant results at the time the study was designed, but these did impact recruitment children living with adults with discordant INH susceptibility results were not enrolled due to the potential benefit of INH preventive therapy. At all sites, recruitment took place over large areas, in poor communities and with over-worked routine healthcare workers, who referred patients to mul- 


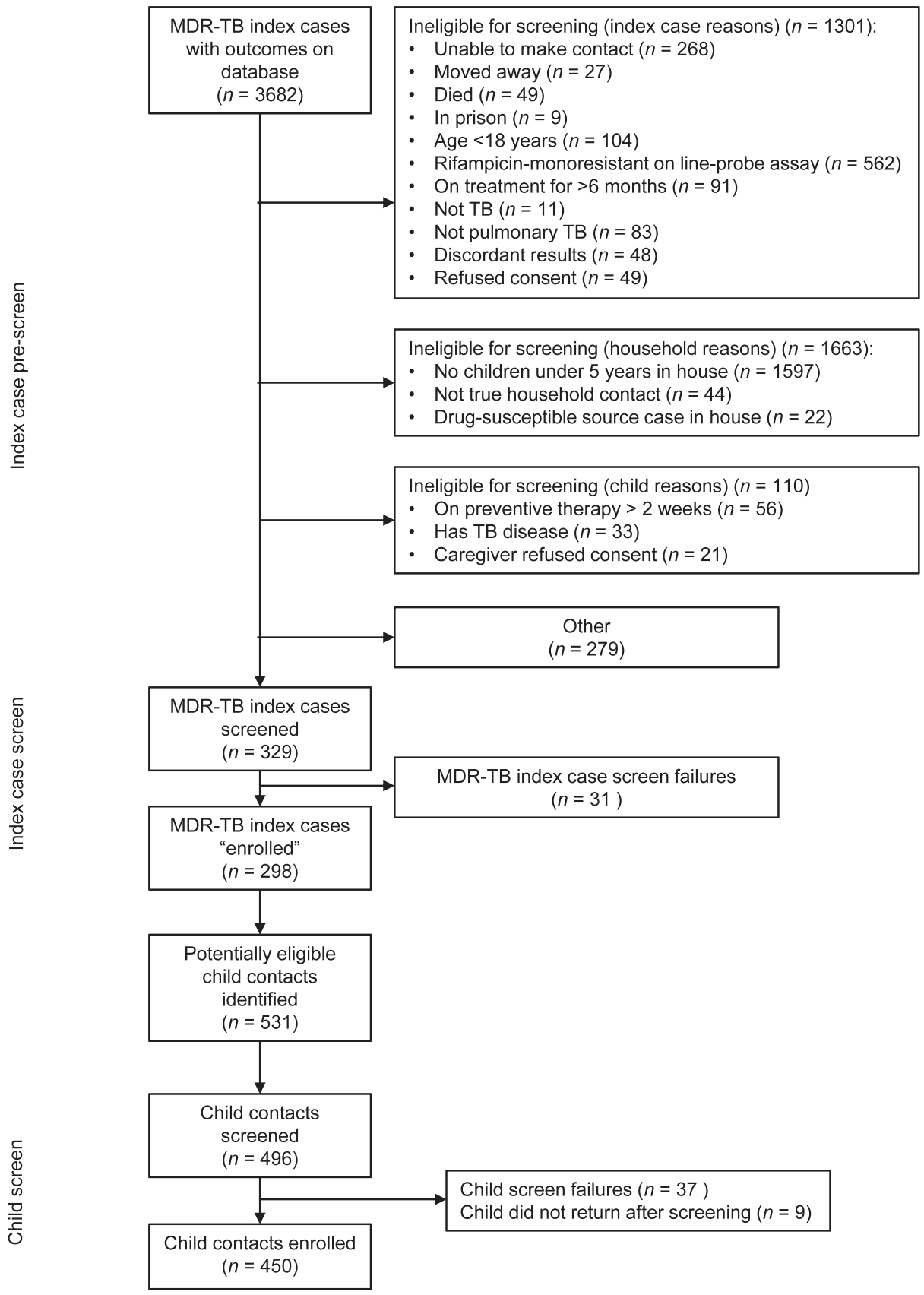

Figure 3 CONSORT diagram showing number of index cases pre-screened and screened, and number of children screened and enrolled on the TB CHAMP trial between 1 September 2017 and 31 July 2019. MDR-TB = multidrug-resistant TB; CONSORT = Consolidated Standards of Reporting Trials; TB-CHAMP $=$ Tuberculosis Child Multidrug-resistant Preventive Therapy Trial.

tiple studies. Recruiters needed extensive local knowledge and spent a considerable amount of time gaining the trust of healthcare workers from numerous clinics and hospitals. In some instances, study vehicles were targeted for violent attacks as they were mistaken for taxis operating during strike action.

\section{Study teams}

Recruitment was found to be very resource-intensive in terms of personnel and vehicles. This meant that teams needed to plan carefully. Personnel needed to have dual roles in some instances. Development of a recruitment tracking system was found to be very beneficial in enabling rapid communication between teams and in avoiding duplication of effort.

\section{DISCUSSION}

Recruitment to prevention trials with rigorous study designs, which focus on infectious diseases and target highly vulnerable populations, is particularly chal- 
Table 1 Participant challenges and solutions to recruiting for a large Phase 3 MDR-TB preventive therapy trial

\begin{tabular}{|c|c|c|}
\hline Challenge & Possible solutions & Implemented? \\
\hline \multicolumn{3}{|c|}{ Adult MDR-TB index case and/or caregiver } \\
\hline $\begin{array}{l}\text { Difficult to contact/locate (lack of contact details, } \\
\text { migration, work schedule, illness, } \\
\text { hospitalisation, incarceration) }\end{array}$ & $\begin{array}{l}\text { Meet index case at clinic, drive together to home. Record } \\
\text { multiple contact details. Work outside normal office } \\
\text { hours. Obtain permission to recruit in hospitals }\end{array}$ & Yes \\
\hline Illness (making consenting difficult), death & $\begin{array}{l}\text { Be prepared to take consent in hospital, over multiple } \\
\text { days. Allow relative of deceased index case to consent }\end{array}$ & Yes \\
\hline Substance abuse (drugs, alcohol) & $\begin{array}{l}\text { Be prepared to visit home on multiple occasions, } \\
\text { especially early morning }\end{array}$ & Yes \\
\hline $\begin{array}{l}\text { Mistrust regarding research studies; low levels of } \\
\text { research literacy }\end{array}$ & $\begin{array}{l}\text { Well trained recruiters from local communities to take } \\
\text { consent; active Community Advisory Board }\end{array}$ & Yes \\
\hline Stigma, fear of rejection/eviction & $\begin{array}{l}\text { Recruiters to discuss stigma at first contact. Use of } \\
\text { unmarked cars and clothing. Option to use own } \\
\text { transport to get to study site }\end{array}$ & Intermittently \\
\hline Index case is a minor & Allow parent/legal guardian to provide consent & No \\
\hline \multicolumn{3}{|c|}{ Child } \\
\hline $\begin{array}{l}\text { In foster care due to illness/hospitalisation of } \\
\text { caregiver - unable to attend study visits }\end{array}$ & $\begin{array}{l}\text { Take consent from parent/legal guardian. Arrange } \\
\text { transport for child and foster parent for follow-up } \\
\text { visits. }\end{array}$ & Yes \\
\hline \multicolumn{3}{|c|}{ Caregiver } \\
\hline No legal confirmation of guardianship & Assist family to obtain guardianship & Yes \\
\hline Second parent refuses consent & Try to involve both parents in consent process & Yes \\
\hline
\end{tabular}

MDR-TB = multidrug-resistant TB.

lenging, and researchers need to plan such trials carefully and in consultation with funders and community stakeholders.

Recruitment for the TB-CHAMP trial faced numerous obstacles. There were far fewer children under 5 in each household than we anticipated and almost half of all index cases were automatically excluded as a result. RIF monoresistance had more impact than expected, indicative of the evolving drugresistant TB pandemic in South Africa. Although MDR-TB was sometimes confirmed later in index cases (based on culture of sputum samples using phenotypic INH DST), teams needed to act on molecular test results that showed INH susceptibility. Teams had to recruit over wide geographic areas and from many healthcare facilities, which was time- and resource-intensive. Locating MDR-TB index cases was difficult, and teams visited clinics multiple times to make contact. Initial recruitment tracking systems soon became cumbersome and did not facilitate rapid communication between team members at different locations. Direct referrals were fewer than anticipated, and laboratory data extracts became key to contacting index cases. Additional time and energy were invested in ongoing meetings with routine healthcare personnel regarding the trial. Contact tracing and recruiting in poor, sometimes violent communities where TB disease is stigmatised, meant extra resources were expended to ensure that study personnel could work safely and sensitively.

Table 2 Study team/resource challenges and solutions to recruiting for a large Phase 3 multidrug-resistant TB preventive therapy trial

\begin{tabular}{lcc}
\hline Challenge & Possible solutions & Implemented? \\
\hline Short staffed, especially drivers & $\begin{array}{c}\text { Budget carefully for support staff; train drivers as recruiters/vice } \\
\text { versa } \\
\text { Lack of recruitment tracking system }\end{array}$ & Over time \\
Dual roles (as recruiter and research assistant) & $\begin{array}{c}\text { does not need to be complex } \\
\text { Carefully structure team and clarify roles - preferable to have a } \\
\text { dedicated recruiting team }\end{array}$ & Over time \\
Lack of team leadership, clearly defined team & $\begin{array}{c}\text { Recruitment team leader is key hire - motivated individual with } \\
\text { structure }\end{array}$ & Oood administrative, interpersonal skills \\
$\begin{array}{l}\text { Communication between team members, } \\
\text { multiple facilities, and study sites }\end{array}$ & $\begin{array}{c}\text { User-friendly recruitment tracking system; WhatsApp groups; } \\
\text { phones, data, airtime to all team members; dedicated study } \\
\text { phone per study site; good internet connection at study sites }\end{array}$ & Over time \\
$\begin{array}{l}\text { High staff turnover } \\
\text { Trial fatigue }\end{array}$ & $\begin{array}{l}\text { Protocols/material in place for rapid training of new staff } \\
\text { Clear targets; staff incentives (meals, social events, small gifts) }\end{array}$ & Over time \\
\hline
\end{tabular}


Table 3 Study design and setting challenges and solutions to recruiting for a large Phase 3 MDR-TB preventive therapy trial

\begin{tabular}{|c|c|c|}
\hline Challenge & Possible solutions & Implemented? \\
\hline Randomised, placebo-controlled trial & $\begin{array}{l}\text { Carefully explain rationale in simple language; meet } \\
\text { regularly with routine healthcare team to discuss study } \\
\text { rationale }\end{array}$ & Yes \\
\hline Prevention trial & Carefully explain benefits of prevention & Yes \\
\hline Long follow-up period & $\begin{array}{l}\text { Explain rationale for follow-up period and stress that } \\
\text { follow-up in routine care would be similar length }\end{array}$ & Yes \\
\hline Time-consuming consent process & Use of recruiters to consent; drivers function as recruiters & Yes \\
\hline $\begin{array}{l}\text { Dual written consent (index case and caregiver) } \\
\text { needed }\end{array}$ & Use of recruiters to consent; drivers function as recruiters & Yes \\
\hline $\begin{array}{l}\text { Index case criteria (adult, MDR-TB, diagnosed } \\
\text { from sputum during last } 6 \text { months, rifampicin } \\
\text { monoresistance excluded) }\end{array}$ & $\begin{array}{l}\text { Data extract from laboratory very useful to identify newly } \\
\text { diagnosed pulmonary TB adult index cases; careful } \\
\text { follow-up and tracking to exclude rifampicin mono- } \\
\text { resistance }\end{array}$ & Yes \\
\hline $\begin{array}{l}\text { Child inclusion criteria (under } 5 \text {, close household } \\
\text { contact, preventive therapy }<2 \text { weeks) }\end{array}$ & $\begin{array}{l}\text { Plan for large recruiting area; attempt to enrol children as } \\
\text { soon as possible after index case is diagnosed }\end{array}$ & Over time \\
\hline Potential duplication of work with routine care & $\begin{array}{l}\text { Develop and pilot good communication tools between } \\
\text { study and routine care }\end{array}$ & Over time \\
\hline Long waiting times during study visits & $\begin{array}{l}\text { Optimise clinic flow with available resources; participant } \\
\text { appointments in different time slots; doctors start day } \\
\text { by writing scripts to avoid pharmacy delays }\end{array}$ & Over time \\
\hline $\begin{array}{l}\text { Migrant population - moving regularly between } \\
\text { homes, suburbs, provinces }\end{array}$ & $\begin{array}{l}\text { Constantly update contact details; anticipate multiple } \\
\text { attempts to make contact }\end{array}$ & Yes \\
\hline $\begin{array}{l}\text { Poor communities (homes difficult to locate, low } \\
\text { level of education, comorbidities, substance } \\
\text { abuse) }\end{array}$ & $\begin{array}{l}\text { Make use of local knowledge, employ staff from local } \\
\text { communities, simple language in study material }\end{array}$ & Yes \\
\hline Violent communities & $\begin{array}{l}\text { Staff safety is paramount: recruiters work in pairs, drivers } \\
\text { accompany recruiters to homes, drivers with advanced } \\
\text { driving skills, avoid potential hotspots }\end{array}$ & Yes \\
\hline Over researched communities & $\begin{array}{l}\text { Ensure excellent synergy and co-operation with other } \\
\text { researchers in the area }\end{array}$ & Mostly \\
\hline Large recruiting area, numerous clinics & Budget appropriately for transport costs & Over time \\
\hline $\begin{array}{l}\text { Health care worker concerns regarding study } \\
\text { design }\end{array}$ & $\begin{array}{l}\text { Face to face contact sessions with healthcare workers, as } \\
\text { well as presentations at clinical meetings, forums; } \\
\text { ready availability of supporting study documentation - } \\
\text { simple, widely distributed }\end{array}$ & Yes \\
\hline $\begin{array}{l}\text { Over-worked health care workers in routine care; } \\
\text { few referrals }\end{array}$ & $\begin{array}{l}\text { Ensure referral to study is not onerous, study decreases } \\
\text { workload for healthcare workers; promotional } \\
\text { materials (mugs, pens, rulers) as reminders of study }\end{array}$ & Yes \\
\hline $\begin{array}{l}\text { Rapid turnover of health care workers in routine } \\
\text { care }\end{array}$ & $\begin{array}{l}\text { Regular updates, posters in each clinic, be prepared to } \\
\text { explain study at each clinic visit }\end{array}$ & As far as possible \\
\hline Conflicting trials & Large recruiting area, develop synergies, cross-referral & Yes \\
\hline $\begin{array}{l}\text { Hospitals and in-patients difficult to locate, often } \\
\text { already discharged }\end{array}$ & $\begin{array}{l}\text { Track index cases to local clinics using address details or } \\
\text { laboratory system; knowledge of local geography and } \\
\text { referral patterns crucial }\end{array}$ & Yes \\
\hline $\begin{array}{l}\text { Frequent unrest/strike action (cars mistaken for } \\
\text { taxis) }\end{array}$ & $\begin{array}{l}\text { Study vehicles to be clearly marked, using magnetic } \\
\text { labelling (removable where stigma is a concern) }\end{array}$ & Yes \\
\hline
\end{tabular}

MDR-TB $=$ multidrug-resistant TB.

In a systematic review of discontinued trials, an overestimated prevalence of eligible participants was the most frequently reported reason for recruitment failure. ${ }^{7}$ Based on our experience, we recommend feasibility studies where possible, but at least the investment of time and resources on a detailed and careful recruitment plan, using current demographic and healthcare data to accurately estimate the prevalence of populations of interest. Nesting additional qualitative work to systematically investigate recruitment challenges and solutions is also recommended. We did not anticipate having to pre-screen 9-10 index cases for each child participant enrolled on TB-CHAMP. This had important implications for our study timeline and funding.

Recruitment strategies need to be dynamic and flexible. Key to this is a tracking/monitoring system that can provide rapid feedback. ${ }^{22}$ Recruiting teams need to be able to track referrals and attempts to contact participants and communicate these effectively. Teams must also be able to plan and strategize based on current data and projections. We found that a customised tracking database was useful and recommend that it be in place before a trial opens. Using trained research assistants and drivers as recruiters frees up clinical personnel. Strong leadership was required to ensure good recruitment and dynamic, organised individuals need to lead recruiting. Constant positive feedback was important, given the discouraging experiences while recruiting.

Our study had limitations. At one site, outcome data were incomplete, influencing our ability to provide a true reflection of reasons why index cases screened out. Challenges and solutions listed are the 
combined opinions of all study personnel members but were not systematically collected. Nevertheless, we have learned invaluable, practical lessons which are relevant to other TB prevention trials.

\section{Acknowledgments}

The authors would like to acknowledge the entire TB-CHAMP team at all three sites for their tireless recruiting efforts and for freely sharing their challenges and experiences; Z Munch from the Geography Department at Stellenbosch University, Tygerberg, South Africa, for the map, clinical personnel from routine TB services at all three sites and all our participants and their caregivers.

JAS is supported by a Clinician Scientist Fellowship jointly funded by the UK Medical Research Council (MRC; London, UK) and the UK Department for International Development (DFID) under the MRC/DFID Concordat agreement (MR/R007942/1). $\mathrm{ACH}$ is supported by a South African National Research Foundation SARChi Chair in Paediatric Tuberculosis. TB-CHAMP is funded by Joint Global Health Trials Scheme of the DFID, the Wellcome Trust (London, UK) and the UK MRC (Grant number MR/M007340/1), the South African MRC (Pretoria, South Africa) and UNITAID though the BENEFIT Kids Project.

Conflict of interests: none declared.

\section{References}

1 Dodd PJ, Sismanidis C, Seddon JA. Global burden of drugresistant tuberculosis in children: a mathematical modelling study. Lancet Infect Dis 2016; 16(10): 1193-1201.

2 Jenkins HE, et al. Incidence of multidrug-resistant tuberculosis disease in children: Systematic review and global estimates. Lancet 2014; 383(9928): 1572-1579.

3 World Health Organisation. Global tuberculosis report, 2020. Geneva, Switzerland: WHO, 2020. https://apps.who.int/iris/ bitstream/handle/10665/336069/9789240013131-eng.pdf. Accessed February 2021.

4 World Health Organisation. Latent tuberculosis infection: updated and consolidated guidelines for programmatic management. Geneva, Switzerland: WHO, 2018. https:// www.who.int/tb/publications/2018/latent-tuberculosisinfection/en/. Accessed April 2018.

5 Sully BGO, Julious SA, Nicholl J. A reinvestigation of recruitment to randomised, controlled, multicenter trials: a review of trials funded by two UK funding agencies. Trials 2013; 14(1): 1-9.

6 Kasenda B, et al. Prevalence, characteristics, and publication of discontinued randomized trials. J Am Med Assoc 2014; 311(10): 1045-1051.
7 Briel M, et al. A systematic review of discontinued trials suggested that most reasons for recruitment failure were preventable. J Clin Epidemiol 2016; 80: 8-15.

8 Patel MX, Doku V. Challenges in recruitment of research participants. BJPsych Adv 2003; 9: 229-238.

9 Albrecht TA, Taylor AG. No stone left unturned: challenges encountered during recruitment of women with advanced ovarian cancer for a phase I study. Appl Nurs Res 2013; 26(4): 245-250.

10 Schulz K, Grimes D. Essential concepts in clinical research: randomised controlled trials and observational epidemiology. $2^{\text {nd }}$ ed. London, UK: Elsevier Health Sciences, 2019: p 109.

11 Kaur G, et al. A survey of facilitators and barriers to recruitment to the magnetic trial. Trials 2016; 17(1): 1-10.

12 Caldwell PH, et al. Clinical trials in children. Lancet 2004; 364 : 803-811.

13 Prinz RJ, et al. Recruitment and retention of participants in prevention trials involving family-based interventions. Am J Prev Med 2001; 20(1): 31-37.

14 Fox GJ, et al. Levofloxacin versus placebo for the treatment of latent tuberculosis among contacts of patients with multidrugresistant tuberculosis (the VQUIN MDR trial): a protocol for a randomised controlled trial. BMJ Open 2020; 10(1): 1-10.

15 Gupta A, et al. Feasibility of identifying household contacts of rifampin- and multidrug-resistant tuberculosis cases at high risk of progression to tuberculosis disease. Clin Infect Dis 2020; 70(3): 425-435.

16 Seddon JA, et al. Levofloxacin versus placebo for the prevention of tuberculosis disease in child contacts of multidrug-resistant tuberculosis: study protocol for a phase III cluster randomised controlled trial (TB-CHAMP). Trials 2018; 19(1): 1-11.

17 Statistics South Africa. Men, women and children: findings of the living conditions survey, 2014/2015. Report No. 03-10-02 (2014/15). Pretoria, South Africa: SSA, 2015. http://www. statssa.gov.za/publications/Report-03-10-02\%20/Report-0310-02\%202015.pdf. Accessed April 2021.

18 Schaaf HS, et al. Evaluation of young children in contact with adult multidrug-resistant pulmonary tuberculosis: a 30-month follow-up. Pediatrics 2002; 109(5): 765-771.

19 Seddon JA, et al. Management of children exposed to multidrug-resistant Mycobacterium tuberculosis. Lancet Infect Dis 2012; 12(6): 469-479.

20 Schaaf HS, et al. Trends in drug resistance in childhood tuberculosis in Cape Town, South Africa. Pediatr Infect Dis J 2020; 39(7): 604-608.

21 Seddon JA, et al. The evolving epidemic of drug-resistant tuberculosis among children in Cape Town, South Africa. Int J Tuberc Lung Dis 2012; 16(7): 928-933.

22 Bjornson-Benson WM, et al. Monitoring recruitment effectiveness and cost in a clinical trial. Control Clin Trials 1993; 52-67. 
CONTEXTE : Il peut être difficile de recruter des patients pour des essais cliniques randomisés, et la lenteur du processus de recrutement peut avoir de graves conséquences. Cette étude avait pour objectif de résumer et d'analyser les défis liés à l'inclusion de jeunes enfants dans un essai de prévention de la TB multirésistante (MDR-TB) en Afrique du Sud.

MÉTHODES : Le suivi du recrutement pour l'essai TBCHAMP se fait à l'aide d'une plateforme de recrutement électronique, qui a été utilisée pour générer un diagramme de flux du recrutement. Les questionnaires structurés individuels, les comptes rendus de réunions et les notes des ateliers de travail ont été thématiquement analysés afin d'identifier les obstacles ainsi que des solutions.

RÉSULTATS : Sur $3682(85,3 \%)$ cas index adultes de résistance à la rifampicine avec résultats avant dépistage, $1597(43,4 \%)$ ont rapporté n’avoir aucun enfant de moins de 5 ans au sein de leur foyer et 562 $(15,3 \%)$ étaient des cas de mono-résistance à la rifampicine. Plus de neuf cas index ont été prédépistés pour chaque enfant inclus. De nombreux obstacles au recrutement ont été identifiés. Une organisation minutieuse du recrutement, des systèmes de suivi des données personnalisés, une équipe dédiée au recrutement bien encadrée, des ressources adéquates pour recruter sur de vastes zones géographiques et d'excellentes relations avec les services de lutte contre la TB de routine se sont révélés être des facteurs clés d'un processus de recrutement réussi.

CONCLUSION : Il peut être difficile de recruter des enfants pour des essais cliniques de prévention de la MDR-TB. Plusieurs essais de prévention de la MDR-TB sont en cours, et les enseignements tirés de l'essai TBCHAMP leur seront pertinents, ainsi qu'à d'autres études de prévention de la TB. 